\title{
ERRATUM
}

\section{Clinical characteristics of and treatment protocol for trapped temporal horn following resection of lateral ventricular trigone meningioma: a single-center experience}

TO THE READERSHIP: An error appeared in the article by Lin et al. (Lin Z, Wang C, Gao Z, et al: Clinical characteristics of and treatment protocol for trapped temporal horn following resection of lateral ventricular trigone meningioma: a single-center experience. $\mathrm{J} \mathrm{Neu}$ rosurg [epub ahead of print February 15, 2019; DOI: 10.3171/2018.11.JNS182710]).

In the Characteristic column of Table 3, "Mean FU duration (mos)" should have been "Mean FU duration (yrs)". The updated table is shown below.
The article has been corrected online as of April 5, 2019.

Zhiqin Lin, MD

Longyan First Hospital, Fujian Medical University, Fujian, China

Zhongli Jiang, MD

Beijing Tiantan Hospital, Capital Medical University, Beijing, China

CORRESPONDING ARTICLE See pp 481-490.

INCLUDE WHEN CITING

Published online April 5, 2019; DOI: 10.3171/2019.3.JNS182710a.

CAANS 2020, except where prohibited by US copyright law

TABLE 3. Comparison of clinical features between surgical and nonsurgical cohorts

\begin{tabular}{|c|c|c|c|c|}
\hline \multirow[b]{2}{*}{ Characteristic } & \multicolumn{2}{|c|}{ Management } & \multirow[b]{2}{*}{$\mathrm{p}$ Value } & \multirow[b]{2}{*}{ All Patients } \\
\hline & Surgical & Nonsurgical & & \\
\hline No. of cases & 11 & 8 & & 19 \\
\hline Mean age (yrs) & $36.7 \pm 14.7$ & $44.3 \pm 12.0$ & 0.282 & $39.9 \pm 13.8$ \\
\hline Female sex & $7(63.6)$ & $6(75.0)$ & 0.494 & $13(68.4)$ \\
\hline Left hemisphere & $6(54.5)$ & $7(87.5)$ & 0.177 & $13(68.4)$ \\
\hline Time from op to onset (mos) & $2.9 \pm 3.3$ & $3.7 \pm 2.9$ & 0.264 & $3.2 \pm 3.0$ \\
\hline Delayed onset of TTH & $6(54.5)$ & $7(87.5)$ & 0.177 & $13(68.4)$ \\
\hline Mean KPS score at onset & $30 \pm 25.7$ & $82.5 \pm 8.9$ & 0.001 & $52.1 \pm 33.3$ \\
\hline Neurological deficit & $5(45.5)$ & $5(62.5)$ & 0.650 & $10(52.6)$ \\
\hline Intracranial hypertension & $9(81.8)$ & 0 & 0.001 & $9(47.4)$ \\
\hline Severe PVBE & $10(90.9)$ & $5(62.5)$ & 0.262 & $15(78.9)$ \\
\hline Mean midline shift (mm) & $9.0 \pm 3.8$ & $2.0 \pm 2.4$ & 0.001 & $6.0 \pm 4.8$ \\
\hline Mean TTH vol at onset $\left(\mathrm{cm}^{3}\right)$ & $39.6 \pm 27.5$ & $25.2 \pm 13.3$ & 0.2 & $33.5 \pm 23.3$ \\
\hline Mean FU duration (yrs) & $4.6 \pm 1.1$ & $5.0 \pm 0.9$ & 0.509 & $4.8 \pm 1.0$ \\
\hline PVBE complete remission & $9(81.8)$ & $3(42.9) \dagger$ & 0.141 & $12(66.7)^{*}$ \\
\hline Mean vol reduction at last FU (\%) & $83.9 \pm 29.1$ & $26.6 \pm 18.8$ & 0.001 & $59.8 \pm 38.1$ \\
\hline Mean KPS score at last FU & $87.3 \pm 12.7$ & $88.8 \pm 9.9$ & 0.863 & $87.9 \pm 11.3$ \\
\hline
\end{tabular}

Values are presented as the number of patients (\%) unless indicated otherwise. Mean values are presented \pm SD.

${ }^{*}$ PVBE was present in 18 patients in the study.

$\uparrow$ A total of 7 patients presented with PVBE in the nonsurgical cohort. 